\title{
Review on-Genome editing tool to combat with multidrug resistant bacteria: Challenges and future perspectives
}

\author{
Ghulam Akbar ${ }^{*}$, Muhammad Anjum Zia ${ }^{1}$, Ali Ahmad ${ }^{2}$, Neha Arooj ${ }^{1}$, \\ Mahpara qamar ${ }^{1}$, Mohsin Raza ${ }^{1}$ and Shahneela Nusrat ${ }^{1}$ \\ 1. Department of Biochemistry, University of Agriculture, Faisalabad, 38000-Pakistan \\ 2. Department of Agronomy, University of Agriculture, Faisalabad, 38000-Pakistan \\ *Corresponding author's email: ghulamakbardgk@gmail.com

\section{Citation} \\ Ghulam Akbar, Muhammad Anjum Zia, Ali Ahmad, Neha Arooj, Mahpara qamar, Mohsin Raza and Shahneela \\ Nusrat. Review on-Genome editing tool to combat with multidrug resistant bacteria: Challenges and future \\ perspectives. Pure and Applied Biology. Vol. 9, Issue 4, pp2441-2455. http://dx.doi.org/10.19045/bspab.2020.90259
}

\begin{tabular}{llll}
\hline \hline Received: 01/04/2020 & Revised: 01/07/2020 & Accepted: 04/07/2020 & Online First: 24/07/2020 \\
\hline
\end{tabular}

\section{Abstract}

These sequences are interspaced short palindromic repeats in regularly clustered manner known as CRISPRs. These sequences are derived from bacteria and archaea and have adaptive immune systems. Modified RNA is used as target for identification of DNA. The nucleic acids are destroyed with Cas enzyme. There are several multidrug resistance bacteria which are gradually increasing it's resistance against most of the present antibiotics. Multidrug resistance bacteria such as Staphylococci and enterococci may cause resistant against almost all antibiotics. Therefore, lives of million' speople are at greater risk due to these multidrug resistant infectious bacteria. Due to lack of novel therapeutic techniques multidrug-resistant bacteria are being out of control with continual increase of their infections. The bacteria and archaea are protected against invading nucleic acids. Therefore, this CRISPR-Cas system is considered as immune system. The bacterial pathogens of clinical significance can be killed by using phage mids (plasmids packed into phage capsids) of CRISPR-cas9 tool. This sequence specific targeting makes CRISPR Cas system unique for the detection of pathogenic and nonpathogenic bacteria. This review describes CRISPR Cas technology, its types, Cas delivery vehicle, mechanism, challenges with their solutions and future perspectives.

Keywords: CRISPR-Cas; Challenges; Delivery; Drug resistance bacteria; Types

\section{Introduction}

The various uses of antibiotics are observed since their discovery in 1929, for the treatments or either to inhibit bacterial infections in case of humans and veterinary medicine. The level of bacterial antibiotic resistance is increased globally so the excess use of antibiotics for prevention and treatment can also be observed [1]. Most of the common infections may remain untreatable due to antimicrobial resistance which exhibits serious threat to modern medicine. Significant efforts are needed in coming years in order to meet the developing approaches to reduce the spread of AMR genes. That's why last decade can be consider as halt in discovery of new antibiotics. United States and Europe show the casualty rate of 
about 50,000 humans per year which is an alarming situation and this number will be significantly increase by the end of 2050 with approximated 10 million deaths per year [2]. This predictednumber of casualtiesdemonstrate the unexceptional similarity with approximately 8.2 million deaths resulting from all types of cancer [3]. Various groups stated the importance of CRISPR-Cas in 2014. It is basically an immune system which consist of clustered regularly interspaced short palindromic repeats interlinked with CRISPR. This tool is found favorable in editing of genomes and can also be utilized for bacterial systems to remove AMR genes in future. Here we are going to discuss about the present condition of this CRISPR-Cas antimicrobials, along with its threats and how they will be solved. World Health Organization (WHO) in 2017 announced the first check list of these bacteria which exhibit antibiotic resistance. Beta-lactam antibiotics were considered to be resistant to the seven pathogens out of the 12 resistant pathogens. In conclusion, the goal for future research is establishing by refocusing on the discovery of new antibiotics [4].

The dispersal of resistance genes can be observed at environmental level due to the excessive and mistreatment of antibiotics which cause resistance in non-pathogenic microbiota, as well as in bacteria to the present antibiotics [5]. There are couple of bacterial species which contribute to escape the action of antimicrobial agents and show the matter of great concern. These species include acronym ESKAPE, Enterobacter spp, Acinetobacter baumannii, Klebsiella pneumoniae, Pseudomonas aeruginosa, Enterococcus spp. and Staphylococcus aureus [6]. The first report of this resistance appearance in India, with its following increase to Pakistan, the United States, Canada, Japan, and the United Kingdom became the evidence for its global status [7].
There are different ways which become responsible for resistance and previously non-existent responses. These ways include insertion of external genes and acquired and selective genetic changes. Recent time show different evolvement of resistance mechanisms. These mechanisms include (change in DNA targets) by DNA gyrase, aminoglycoside $\mathrm{N}$-acetyltransferase for inactivation of fluoroquinolones, DNAbinding proteins (the Qnr family) for protection of targets, aminoglycosides for blockage of the $30 \mathrm{~S}$ ribosomal subunit and exportation of drugs out from microorganisms due to increased efflux [8, 9]. There are some changes which are already reported in antimicrobial agents like chemical structural changes agents [10], changes in permeability of membranes along with alternations in the target of antimicrobial attack [13] and decreased concentration of the antimicrobial at its place of attack $[11,12]$.

The alternations in the cell envelope of $P$. aeruginosa that are connected with resistance to polymyx in $\mathrm{B}$ do not involve the expression of porin which is considered as mechanism of permeability reduction [14]. The phenomenon of tetracycline and macrolides is greatly affected when the cell wall acting antibiotics such as penicillin and glycol peptides cause the alternations in the antimicrobials targets along with the reduce activities of other antimicrobials that act on the bacterial ribosome [15, 16]. Antimicrobials exert selective pressure which leads to natural selection and conclude the influence of several bacterial groups with death of suspectable microorganisms. The number of these mechanism are increasing in large number of species [17].

In 2014, 243 studies were observed by Bell in meta-analysis. These studies stated that "The great resistance can be produced in individuals due to increased utilization of bacterial resistant drugs and this resistance is 
not only limited to individual level but to the area, community and countries, which can create danger to persons". The misuse of antibiotics was investigated worldwide between 2000 and 2010 in another study of the same year. Each around was found to increase the $36 \%$ consumption of antibiotics. But approximately $76 \%$ increase can be observed in case the BRICS group (Brazil, Russia, India, China, and South Africa). The significant increase can be seen in the usability of these drugs rather than their worrying trends to treat the diseases [18]. The direct relationship can be observed between increase in resistance indicators and usage of antibiotics [19]. There is an urgent need to redefine the contact between mankind and contagious illness because still no discovery is observed in case of molecules to combat resistant microorganisms [20].

The bacterial resistance is great matter of concern which should be declared urgently. The genetic determines were discovered from the soil through functional meta-genomic studies. The only one fraction of these genetic determinants has been shown in human pathogens and the antibiotics resistance [21]. So, it is serious requirement to createlatest antibacterial drugs to protect microbiota and to control the spread of antibiotics resistance. The different strategies are highlighted here to be utilized in future to inhibit bacterial resistance. These strategies include CRISPR-Cas9, use of nanotechnology and treatment with bacteriophages nanoparticles. These strategies could be used in combination with integrated strategies as well as to directly combat microorganisms in individuals. The introduction of new antimicrobials is not possible in short duration. Even the scientific community has highlighted that there are no new proposals for new discovery of antibiotics. The techniques for increased activity are combined with classical antibiotics which is considered as recommended approach for rational use. Different techniques and resistance mechanisms like beta-lactamase, efflux pump, and quorum sensing inhibitors, as well as bacteriophages and new drug delivery systems increase the activity mechanism of antibiotics by reducing or blocking these mechanism [22- 25].

Clustered regularly interspaced short palindromic repeats (CRISPRs)

Bacteria and archaea are used to originate adaptive immune systems which are shortly known as CRISPRs. The recognition of DNA target can be done by using modified RNA and destruction of nucleic acids with the help of Cas enzyme. Ultimately, we can say that the binding and cleavage need only one protein. Natural CRISPRs are utilized to develop new molecular instruments based on simplicity of this system as shown in (Fig. 1). The cytotoxicity of these instruments is considered as main feature because of their ability to directly kill bacteria, antimicrobial attack and ability to active immunity in bacteria [26-28]. The selective and particular medical purposes to remove bacteria are achieved with the help of CRISPR-Cas systems. CRISPR Cas systems are causing the development in medication of multidrugresistant infections because they use sequence information to remove single bacterial strains in selective and particular manner. Hence, they are more generalized and customizable techniques. Although some other antimicrobial strategies are also present but they are limited to the partial solutions. Different systems act as antimicrobials and studies show evidence for these systems. The bacterial species and strains can be killed particularly with both heterologous and endogenous systems and this study was reported by Gomaa in 2014. 


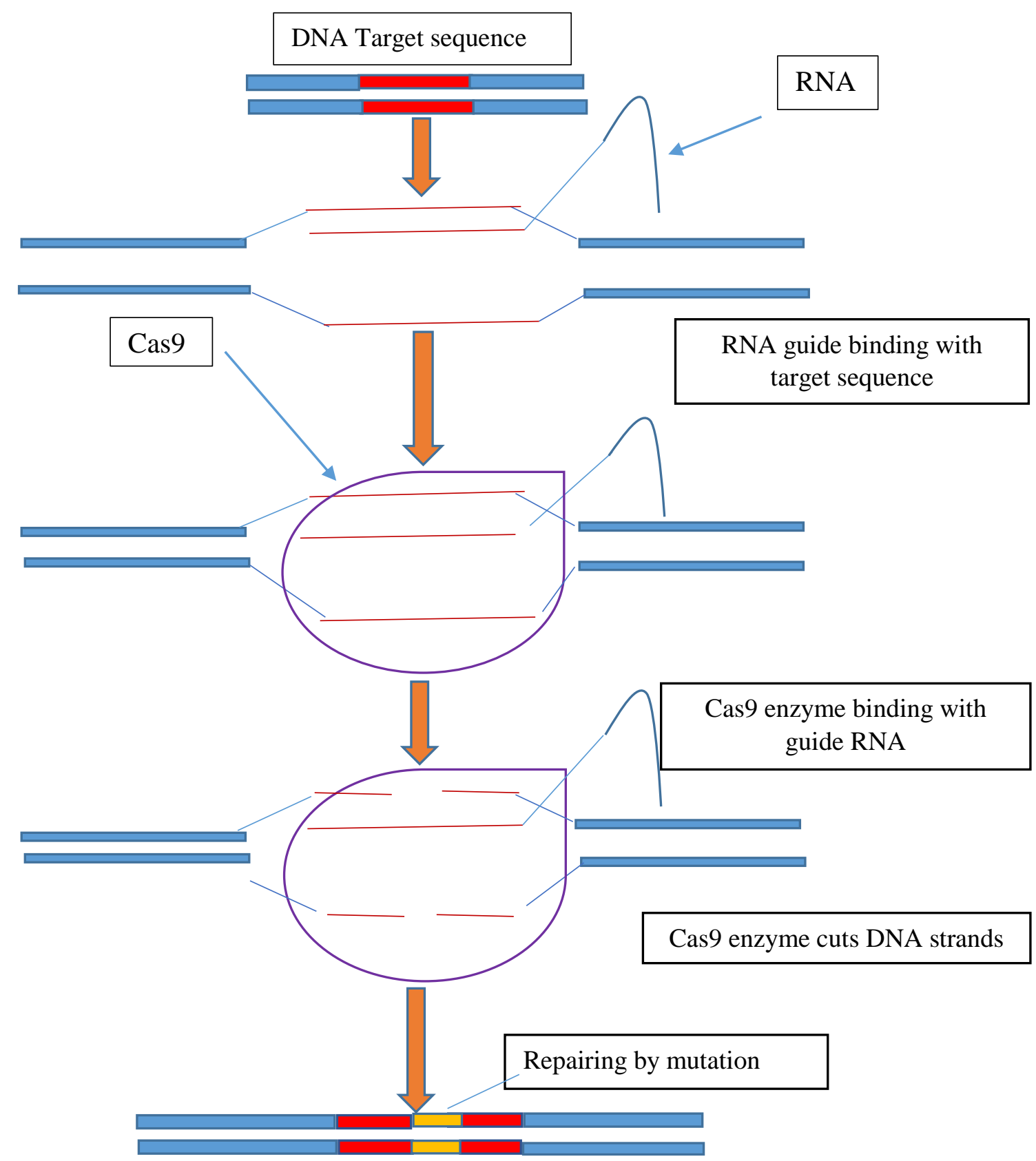

Figure1. CRISPR CAS 9 with its mode of action

The antimicrobial based on CRISPR Cas could cause the cell death at any location of genome and this information was revealed by sequences present on target genome [29]. RNA-guided nucleases (RGNs) allow the remolding of microbiota by targeting the undesirable polymorphisms or specific resistance genes. This usage categorizes in one of the most important use in technology to proceed antimicrobial action [30]. CRISPR Cas are considered to function as precision antimicrobials in the research comtucted by Fuente-Núnez and Lu in 2017. In this study, it was found that this system seemed to be involve in targeting genes of antibiotic resistance, virulence, biofilm formation along with the removal of drug resistant microbes [31]. Although the studies 
show support to this system but there are various issues which need to be solved in order to use them as antimicrobial agents as well as efficient delivery vehicle [32]. Pan performed study in 2017 in order to focus on the issues of CRISPR delivery and passages. He used multi-host bacteriophage K64-1 to identify eight depolymerases and then nine capsule depolymerases from K64dep (S2-5) after characterization [33].

The bacteriophages as carriers are still an issue for CRISPRs system. The use of CRISPR target was obtained by the alternation in the genome of Klebsiella bacteriophage. This study was perfomed by shen and his colleague scientists in 2018 and aimed to give positive results [34]. The desired specificity can be achieved through nanotechnology. This nanotechnology can be used for CRISPRs delivery and it can also give alternations on surface [35]. There is still matter of great concern to use CRISPR Cas in future research for its safe usability and attacking resistant microorganism pointed out by Pursey in 2018. The resistance of bacteria oppose CRISPR Cas is also matter of concern because of native procedures existence [36]. Resistance genes in bacteria reduce the activity of natural CRISPR-Cas and this information was confirmed. He conducted research on multidrug-resistant Shigella which proved to provide great knowledge. The safe delivery and evaluation of present resistance technology demands an integrative research where there should be proper cooperation of scientists for sequences study [37]. The virulence and resistance are an alarming situation in bacteria because it leads to the adaptation. Bacteria have ability to store different combinations and genes of resistance and toxicity proved by various studies. KPC-2 gene showed the adaptation to different mobile elements and this study was performed by [38].

This study paved the way to evaluate for various applications of this CRISPR system
[38]. Two new DNA editing systems were also introduced through the research performed on carbapenem-resistant $K$. pneumoniae. Genome editing will show facilitation to consider treatments for resistance to carbapenems. Plasmid pCasKPpSGKP and plasmid system pBECKP were observed to be excellent in genome editing [39]. Hullahalliin 2017, 2018 performed studies on Enterococcus faecalis and this organism showed the tolerance of CRISPRCas with the genetic basis of phenotypes. Although, a lot of studies were performed against multidrug resistant bacteria but this study paved the way in better understanding for dealing problems created by the use of CRISPRS and response of organisms. As result of this knowledge, tolerance level of different phenotypes was also studied [40]. Hence this study will help researchers to study genomic and metabolic pathways of resistant bacteria and new techniques will help researchers to attack resistant bacteria so that resistance issues will not happen in future.

\section{Types of CRISPR-Cas system}

There are 3 sorts of CRISPR-cas system. In 1987, meanwhile research of theiap gene, Nakata and his friends demonstrated a group of nucleotides which is repeated in $E$. coli, which was beginning of story of CRISPR-cas system [41]. In the next decades, by arranging the sequence of numerous microbial genomes such as archeal and bacteria strain, many repeat elements from the genome were reported [42]. After some time, family of this unique inter spacing repeat sequencing were named as clusters of repeated elements. The name CRISPR was demonstrated by [43]. By suggesting the phase association or extrachromosomal origins [44, 45] spacer sequences were isolated from their direct repeats, it was done in 2005. The fundamental functional units and processing of CRISPR has been 
cleared in 2010. That system consists of spacer sequences, locus of gene with unrepetitive and side by side 6-20 genes that are able to read CRISPR proteins $[46,47]$. CRISPR-cas system has been utilized in different approaches related to biotechnology as well as for the generation dairy cultures which show resistance towards phage [43].

In bacteria and archea CRISPR-cas system is an adaptive immune system that saves bacteria from invades such as bacteriophages/phages andMGEs (mobile genetic elements) [48]. This system destroys the foreign genetic element in 3 phases. Adaptation or spacer acquisition is considered as $1^{\text {st }}$ phase [49], in which after recognition spacer sequence is indulged into CRISPR arrangement. The $2^{\text {nd }}$ phase is expression of crRNA (CRISPR RNA) or biogenesis, in that stage RNA polymerase transcribed the pre-CRISPR RNA (pre-crRNA). Special endonucleases cleaved these pre-crRNA into into small crRNA. On the basis of crRNAs functions, these are also known as guide RNAs $[50,51]$. The final or third stage is the interference, [52] in which perfect complementarity with foreign RNA or DNA base pair is recognized and formed by crRNAs [46, 47]. This step goes ahead to the breakdown of crRNA out sided complex of nucleic acids. On the other hand, the host is susceptible to infection if cleavage does not occur. This is may be due to any disterbance in PAM(proto-spacer adjacent motif) or may be dissimilarcombination of spacer and DNA of invader [53].

CRISPR-cas system has been classified into following groups:

CRISPR-cas system I

CRISPR-cas system II

CRISPR-cas system III

The presence of signature genes in all types is the reason for this classification.
For instance, type I has cas3 gene, type II has cas9 gene and type 3 has cas 10 gene. Thespacer show significance with the contribution of protein 1 and protein 2 [46, 47, 53]. Therefore, each types and subtypes of CRISPR system consist these proteins. Different species of bacteria have different types of CRISPR-cas.

\section{Type I system}

In most bacteria and archaea, CRISPR-cas system is present[54]. Six sub-groups (A-F) are formed of this system, which encodes cas 3 gene. This Cas 3 protein is a multidomain protein, with the property of nuclease and helicase activity [55]. There are two domains of this protein: an N-terminal HD phophohydrolase for the breakdown of DNA molecules and second is C-terminal DExH helicase domain which unwinds DNA winded strand $[46,47]$. These two domains work together and performed the degrading of DNA of invader. Anyhow, only cas3 is unable to recognize the DNA of invader and safe cell from infectious particles $[51,56]$. A number of plex was also seen in S.solfataricus, [57] in each subtype of type I CRISPR-cas system. Moreover, crRNA guided surveillance complexes have been recognized in Bacillus halodurans (type I-C) [58] and Pseudomonas aeruginosa (type I-F) [59].

\section{Type II system}

Bacteria contain only type II system, which is simplestas compared to other types. [54, 53]. Type II has four numbers of genes: cas 1 gene, cas 2 gene, cas 9 gene and cas 4 gene in the case of type II-B or $\operatorname{csn} 2$ in type II-A case. The major protein of type 2 is cas9, it plays a great part in crRNA biogenesis as well as in breakdown of DNA of invader [60].The cas9 has two domains in which one is RuvC domain and other is HNH domain. The function of RuvC domain is cleavage of non-complement strand, whereas the $\mathrm{HNH}$ domain involved in 
cleavage of DNA which is complement to crRNA guide. [61]. In typeII system, for the biogenesis of crRNA transactivating crRNA (transcrRNA) is required. Reading of tracrRNA in Streptococcus pyogenes is held against the CRISPR-cas locus strand [47]. The construction of double stranded RNA is made by the hybridization of crRNA repeats and tracRNA, which is recognized and ruined by the cellular non-cas RNase III enzyme. The elimination of cas9 gene causes the inhibition of biogenesis of crRNA, however, role of cas 9 is still unclear in biogenesis [60]. It has been demonstrated by Jinek and his workers, that cas9 enzyme require both crRNA as well as tracr RNA to destroy the targeting DNA [61]. All the domains which are required for the DNA breakdownseem to be joined in one protein (cas9), which enables the type II system as unique and best selection for genome [62].

\section{Type III system}

There are two sub-groups of type III system: type III-A and type III-B [54]. Commonly, archaea consist this system. Type III-B ispresent in combination with different other types of CRISPR. This system encodes cas6 gene as well as cas10 gene [63]. Cas10 is also termed as repeat associated mysterious protein (RAMP). It highly takes part in maturation of crRNA as well as in cleavage of DNA [63]. Cas6 is an endonuclease, works independently and is not associated with CASCADE complex [64]. In addition, cas6 may be present in archaea, which also have type III system along with CRISPR-cas, type I-A or type I-B [65].

Type III CRISPR-cas system subtypes have many similarities but these subtypes target the substrates which are chemically different. For example, type III-A system S.epidermis targets DNA, on the other hand type III-B system present in $S$. solfataricus and Pyrococcus furiosus [46, 47] act on RNA. This claims the diversity of CRISPR system present within the type III system.

Targeting of antimicrobial resistance in bacteria by CRISPR-Cas

The attack of nucleic acids is prohibited when immune system of CRISPR-Cas provide protection to bacteria and archaea against it. Foreign bacteriophages and plasmids have DNA or RNA which are present on the CRISPR loci of bacterial genome and use further to identify and attack the nucleic acids carrying the same sequence by the CAS protein machinery. There are six types and six classes of CRISPR-Cas systems. The steps for cleavage and foreign DNA identification are carried out by types I, III, and IV belonging to class 1 and they have very complex architecture. On the other hand, single multidomain recognition and cleavage are performed by types II, V, and VI belonging to class 2 systems with very simple architecture. The revolution in ecological engineering and genome editing are made possible by type II CRISPR-Cas9 system because of its specificity, versatility, and simplicity in targeting. One of these applications has received little attention because of its lower efficiency to remove AMR genes from bacterial populations and communities after reviewing applications [66]. It was postulated many times ago that specific bacterial genotypes could be killed by using synthetic CRISPR-Cas system as an antimicrobial [67].

This prospective of CRIPSR-cas has been confirmed by many studies to detach AMRencoding plasmids. These plasmids are used to remove the bacterial strains that consist genes of drug resistance from communities. They can also be used to re-sensitize bacteria to antibacterial drugs, the specificity of CRISPR-cas was revealed when experiment was performed on genotypes of mixed 
population of Escherichia coli to remove separate bacterial strains. Then each unique sequence genotype was targeted with a programmed plasmid encoding CRISPRCas. Clinically related bacterial pathogens $E$. coliand Staphylococcus aureuscould be killed by CRISPR-cas using plasmids packed in phage capsids and this information was demonstrated in two studies. The targeting of AMR genes on plasmids were approached by using phagemid transduction to deliver CRISPR-Cas9 in one study. Hence, this approach was proved very efficient to remove these plasmids from bacteria. Bacteria carrying chromosomal AMR genes were effectively killed by these conjugative plasmids of CRISPR-Cas9. Phagemidmediated delivery of CRISPR-Cas9 system was confirmedefficient to remove plasmids carrying AMR genes in other study. The removal of desensitizing bacteria to antibiotics were also confirmed in this study [28-30].

It was also revealed in these studies that specific bacteria could be killed in vivo either the larvae of Galleria mellonella disclose to enterohaemorrhagic $E$. colior with the surface of mice which were colonized with S. aureus. But the usability of this system for cure purposes cause that the bacteria could be re- sensitized to antibiotics but this issue allows residual resistant bacteria in order to maintain in the population and bacteria was not able to get benefit over resistant ones.Temperate and lytic phagefound to be used in technology which was developed in order to re-sensitize bacteria to $\beta$-lactam medicines and to increase the advantage of re-sensitized bacteria. In this technology, temperate phage is utilized to target AMR genes by CRISPR-cas. The benefit to resensitized bacteria was achieved through CRISPR-cas using lytic phage [68]. For broad-spectrum targeting CRIPSR-cas was used on common -lactamase gene of $E$. coli in another study and this study indicated the high sequence diversity issues among $\beta$ lactamase genesby the identification of shared target sequence in >200 mutational variants of this gene $[69,70]$.

\section{The architecture and delivery of CRISPR- Cas}

Bacterial species show diversity in case of AMR genes which are present on plasmids and utilize horizontal gene transfer to create diversity [71]. The delivery of CRISPR-cas can be done by phage vectors but there is a hurdle to use them as targeting various bacterial species because of their limited range of hosts. This approach will further add a challenge. The spatial structure and complex microbial communities decrease the attacking rates and this problem will be overcome by engineering phages to increase ranges of hosts [72] but this technology is at initial phases. There is also another technology to use conjugative plasmids as delivery vectors but there are also some issues like restricted ranges of hosts, Conjugation efficacyand hurdles in plasmids uptake and establishment [71]. The cytotoxicity can disturb the effectiveness of CRISPR-cas in some species.Therefore, it is important to consider the efficacy of CRISPR-cas for specific hosts. There some examples which show the toxicity of CRISPR cas9 [73] like Synechococcuselongatus, a cyanobacterium and the toxicity of nucleases [74] in Corynebacterium glutamicum. But alternative class 2 of nuclease Cas 12 a can be use efficiently.

\section{CRISPR-Cas to overcome the dangers of antibiotics resistance}

DNA binding proteins are classified into four classes which are used to edit genome. For example, bacteria (Xanthomonas) are used to produce transcription activator like effectors (TALEs), eukaryotic transcription factors give rise to Zinc finger nucleases (ZFNs)microbial MGEs give rise to mega- nucleases $[43,75,76]$ 
and at final stage, the CRISPR-cas type II system of bacteria give rise to DNA endonucleases cas9 [62]. The low sequence specificity of targeting DNA is the reason for not doing the genome editing by mega nucleases [43]. The building of desired sequence and limited target selection makes ZFN less desirable. The high specificity of targets is achieved in case of TALENs because they are easy to design and have longer DNA binding protein domains. The TALEN cannot deliver in to cells due to their larger size they tend to produce complications as compared to ZFN [77].

Resistance evolution against CRISPR-Cas Evolution for the resistance of CRISPR-Cas is one of the major problems. When the interactions between CRISPR and phage are considered, it is think that due to point mutations in the target CRISPR-Cas sequence, evolution of resistance is done [78]. Basically, this can be occurred in AMR genes which are pointed for removal, especially the genes that are given positive results (which includes the antibiotics containing AMR genes for resistance). On the other hand, resistance can be obtained by making addition or exertion in cas genes, which are necessary for target breakdown, or by removing the target spacers $[79,80]$. It makes the CRISPR-Cas loci inactive. It has been reported that antimicrobials based on CRISPR-Cas9 have less mutations in the target sequences as compared to the defective CRISPR system delivery [81, 82]. The phenomena of the resistance is also said to be evolved from anti-CRISPR (acr) genes, other than the evolution due to the mutation. These genes are responsible for encoding small protein particles that bind to the important parts of CRISPR-Cas immune system and make it inactive. Now a days, more than twenty families of acr genes have been recognized, which cover the type I as well as type II CRISPR-Cas systems [83, 84]. It has been seen that families of Acr proteins for type I are isolated from phages which are infection Pseudomonas aeruginosa and many other species of peoteobacteria. Many of the Acr proteins are moreparticular for their CRISPR-Cas subtype, but one of them targets two subtypes of CRISPR-Cas which includes typeI-E and type I-F [85]. It shows that broad spectrums of these proteins exist. At present, type II targeting Acr proteins have also been identified, which are responsible for gene editing. Due to highly specific property and large diversity of sequence these Acr proteins are present everywhere and may be transformed by MGEs which includes plasmids as well as phages. Extra research and study are needed for finding the impact of evolutionary results of AMR genes as well as for the dynamics of population. For the knowledge of evolutionary and ecological demands of CRISPR-Cas system, more studies on microbial communities are required [30,67].

\section{Challenges for Complex microbial communities}

CRISPR-Cas system has great ability for killing the sequence which is specific or may be resensitisation of AMR carry bacteria. In the present age, to minimize the AMR genes, CRISPR-Cas has been used in clonal bacterial populations. In the real environmental world, bacteria are typical microorganisms which are more challenging by using this technique [71]. Communities of microbes have a large number of cells per gram of matrix. These communities are taken from animal, human and environmental micro biomass, having various kinds of species. When a single species is considered, bacterial colon may contains many plasmids and MGEs (mobile genetic elements) having different resistance genes. The characterization and quantification of bacteria, mobile genetics elements or resistance genes is held by quantitative PCR (polymerase chain reaction). For finding the 
resistance genes in the complex communities of bacteria, time consuming techniques are used such as fluorescence activated technique and mobile genetic elements prior to downstream analyses.

Other techniques including epic PCR as well as Meta3C are used for determining mobile genes present on specific bacteria and there is no need of cultivation in this process $[30,86]$. One more challenge concerned with community of microbes is the problem in analyzing the community behavior against perturbations. There are unexpected effects of CRISPR-Cas based antimicrobials, which include, if the removal of strain from population or the removal of specific plasmid is done, the growth or metabolic activity is changed, which results in the formation of more potentially active unwanted species. For example, it has been stated that the stress causes alternations in the composition of microbial population, the level of metabolites are associated with enhance sensitivity to Clostridium difficile infections in intestine [67] and therefore transfers in the structure of species of microbiome that are associated with diabetes and periodontitis [71]. There is huge risk of AMR genes destruction with the aid of CRISPR-Cas system in different microbial populations.

\section{Overcoming these challenges}

There are many problems of using CRISPRCas system for AMR genes but there are also solutions to overcome these challenges. The hot issue is to searching of a proper delivery vector for removal of AMR by using the CRISPR-Cas system [86]. For the acute infections, phage vector may be used. However, strains of same bacterial strains provide different vectors. The broad spectrum of hosts of some conjugative plasmids would enable them as a good candidate for use with the combination of probiotic to save from the invasion of AMR gene containing bacteria or to replace AMR reservoirs of microbiome. The suitable cost of conjugative plasmids limits their distribution $[87,88]$. But the mutations or changes in the bacterial host or the plasmid give the advantage of spreading the CRISPRCas plasmid in bacterial groups. If the wide effective host range vector can be isolated or manufactured, the improvement in the delivery system will be occurred and most of the challenges will be solved. In the practical work, the use of CRISPR-Cas based antimicrobials produces unwanted ecological results by the microbial community, which is one of the major problems. Therefore, keen studies are required for determining the effects of removing of AMR genes on different species of bacteria and plasmids. By seeing many applications, the evolution of resistance is impossible to avoid. Although, the resistance by mutations in the sequences can be prohibited by multiplexing, this includes the multiplication of different targeting sequences to overcome the resistance [89]. The use of acr genes can be reduced by using many CRISPR-Cas systems at the same time, which required many Acrs proteins for targeting and manufacturing Acr insensitive CRISPR-Cas system. The use of different nucleases to Cas9, which includes Cas12a, can also avoid many problems such as toxicity and increase the efficiency of system in different hosts of bacteria.

\section{Future prospects}

There are still many problems which need to be solved. For example, the use of CRISPRcas to attack antimicrobial resistance genes in microbe populations. By reprogramming the CRISPR-Cas system and enables the construction of genes of interest, can increase the efficiency of this system. More future studies are required to optimize distribution of CRISPR-Cas in the communities of microbes and to make check and balance of the risks of this technique. The CRISPR-Cas system, naturally present in plasmids, may help to sought out the problems of organisms which are genetically engineered. The 
invention of DNA by CRISPR-Cas on large scales will play a key role for sustainability, as well as a risk-free technology.Selection of an appropriate temperature and vectors such as lytic phages which are specific for particular pathogens is one of the major challenges. Therefore, it is necessary to form universal vectors for effective transfer of DNA into different disease causing agents. Phages containing CRISPR-Cas 9 can be used in strains of non-laboratory, without disturbing indigenous genetic information of microbes. In future, conjugative delivery of antimicrobials from probiotic bacteria may be adaptable via CRISPR-Cas technology with accurate targeting in poly microbial intestinal flora.

\section{Authors' contributions}

Conceived and designed the experiment:G Akbar, MA Zia, A Ahmad, N Arooj, MQamar, M Raza\& S Nusrat, Performed the experiment:G Akbar, MA Zia, A Ahmad, N Arooj, MQamar, M Raza\& S Nusrat, Analyzed the data: G Akbar, MA Zia, A Ahmad, N Arooj, MQamar, M Raza\& S Nusrat, Contributed reagents/ materials/ analysis tools:G Akbar, MA Zia, A Ahmad, N Arooj, MQamar, M Raza\& S Nusrat, Wrote the paper:G Akbar, MA Zia, A Ahmad, N Arooj, M Qamar, M Raza\& S Nusrat.

\section{Acknowledgement}

No financial assistance was obtained for this review paper

\section{References}

1. Ali J, Rafiq QA \& Ratcliffe E (2018). Antimicrobial resistance mechanisms and potential synthetic treatments. Future Sci OA 4: 290-299.

2. Simlai A, Mukherjee K, Mandal A, Bhattacharya K, Samanta A \& Roy A (2016). Partial purification and characterization of an antimicrobial activity from the wood extract of mangrove plant Ceriops decandra. Excli J 15: 103-108.

3. Jansen KU, Knirsch C \& Anderson AS
(2018). The role of vaccines in preventing bacterial antimicrobial resistance. Nat Med 24: 10.

4. Shrivastava SR, Shrivastava PS \& Ramasamy J (2018). World health organization releases global priority list of antibiotic-resistant bacteria to guide research, discovery, and development of new antibiotics. J Med Soc 32: 76.

5. Nitsch OA, Gyrczuk E, Wardyn A, Życinska K \& Brydak L (2015). Antibiotic prescription practices among children with influenza. In Respiratory Contagion 2531.

6. Boucher HW, Talbot GH, Bradley JS, Edwards JE, Gilbert D, Rice LB \& Bartlett J (2009). Bad bugs, no drugs: no ESKAPE! An update from the Infectious Diseases Society of America. Clin Infect Dis 48: 1-12.

7. Rios AC, Moutinho CG, Pinto FC, Del FFS, Jozala A, Chaud MV \& Balcão VM (2016). Alternatives to overcoming bacterial resistances: state-of-theart. Microbiol Res 191: 51-80.

8. Redgrave LS, Sutton SB, Webber MA \& Piddock LJV (2014). Fluoroquinolone resistance: mechanisms, impact on bacteria, and role in evolutionary success. Trends Microbiol 22: 438-445.

9. Kapoor G, Saigal S \& Elongavan A (2017). Action and resistance mechanisms of antibiotics: A guide for clinicians. $J$ Anaesthesiol Clin Pharmacol 33: 300.

10. Alekshun MN \& Levy SB (2007). Bioorg. Med Chem Lett Cell 128: 1037-1050.

11. González BC (2017). Antibiotic adjuvants-A strategy to unlock bacterial resistance to antibiotics. Bioorg Med Chem Lett 27: 4221-4228.

12. Willers C, Wentzel JF, Du PLH, Gouws C \& Hamman JH (2017). Efflux as a mechanism of antimicrobial drug resistance in clinical relevant microorganisms: the role of efflux inhibitors. Expert Opin Ther Ter 21: 2336.

13. Sieradzki K \& Markiewicz Z (2004). Mechanism of vancomycin resistance in 
methicillin resistant Staphylococcus aureus. Pol J Microbiol 53: 207-214.

14. Hao M, Ye M, Shen Z, Hu F, Yang Y, Wu $S$ \& Wang M (2018). Porin deficiency in carbapenem-resistant Enterobacter aerogenes strains. Microb Drug Resist 24: 1277-1283.

15. Falagas ME, Kasiakou SK \& Saravolatz LD (2005). Colistin: the revival of polymyxins for the management of multidrug-resistant gram-negative bacterial infections. Clin Infect Dis 40: 1333-1341.

16. Poehlsgaard J \& Douthwaite S (2005). The bacterial ribosome as a target for antibiotics. Nat Rev Microbiol 3: 870-881.

17. Wu JY, Kim JJ, Reddy R, Wang WM, Graham DY \& Kwon DH (2005). Tetracycline-resistant clinical Helicobacter pylori isolates with and without mutations in $16 \mathrm{~S}$ rRNA-encoding genes. Antimicrob Agents Chemother 49: 578-583.

18. Tello A, Austin B \& Telfer TC (2012). Selective pressure of antibiotic pollution on bacteria of importance to public health. Environ Health Perspect 120: 1100-1106.

19. Van BTP, Gandra S, Ashok A, Caudron Q, Grenfell BT \&Levin SA (2014). Global antibiotic consumption 2000 to 2010: an analysis of national pharmaceutical sales data. Lancet Infect Dis 14: 742-750.

20. Bell BG, Schellevis F, Stobberingh E, Goossens H \& Pringle M (2014). A systematic review and meta-analysis of the effects of antibiotic consumption on antibiotic resistance. BioorgMed Chem Lett14: 13.

21. Hogberg LD, Heddini A, \& Cars O (2010). The global need for effective antibiotics: challenges and recent advances. Trends Pharmacol Sci 31: 509-515.

22. Forsberg KJ, Patel S, Gibson MK, Lauber CL, Knight R, Fierer N \& Dantas G (2014). Bacterial phylogeny structures soil resistomes across habitats. Nat 509: 612616.

23. Moo CL, Yang SK, Yusoff K, Ajat M,
Thomas W, Abushelaibi A \& Lai KS (2019). Mechanisms of antimicrobial resistance (AMR) and alternative approaches to overcome AMR. Curr Drug DiscovTechnol 3: 40-48.

24. Mulani MS, Kamble EE, Kumkar SN, Tawre MS \& Pardesi KR (2019). Emerging strategies to combat ESKAPE pathogens in the era of antimicrobial resistance: a review. Front Microbiol 10: 539.

25. Pham TN, Loupias P, Dassonville KA \& Sonnet P (2019). Drug delivery systems designed to overcome antimicrobial resistance. Med Res Rev 39: 2343-2396.

26. Vikesland P, Garner E, Gupta S, Kang S, Maile MA \& Zhu N (2019). Differential drivers of antimicrobial resistance across the world. Acc Chem Res 52: 916-924.

27. Sorek R, Lawrence CM \& Wiedenheft B (2013). CRISPR-mediated adaptive immune systems in bacteria and archaea. Annu Rev Biochem 82: 237-266.

28. Bikard D, Euler CW, Jiang W, Nussenzweig PM, Goldberg GW, Duportet X \& Marraffini LA (2014). Exploiting CRISPR-Cas nucleases to produce sequence-specific antimicrobials. Nat Biotechnol 32: 1146-1150.

29. Gomaa A, Klumpe HE, Luo ML, Selle K, Barrangou R, \& Beisel CL (2014). Programmable removal of bacterial strains by use of genome-targeting CRISPR-Cas systems. M Bio 5: 928-13.

30. Citorik RJ, Mimee M, \& Lu TK (2014). Sequence-specific antimicrobials using efficiently delivered RNA-guided nucleases. Nat Biotechnol 32: 1141.

31. De LNC \& Lu TK (2017). CRISPR-Cas9 technology: applications in genome engineering, development of sequencespecific antimicrobials, and future prospects. Int 9: 109-122.

32. Beisel CL, Gomaa AA \& Barrangou R (2014). A CRISPR design for nextgeneration antimicrobials. Genome Biol 15: 516.

33. Pan YJ, Lin TL, Chen CC, Tsai YT, Cheng YH, Chen YY \& Wang JT (2017). 
Klebsiella phage $\Phi$ K64-1 encodes multiple depolymerases for multiple host capsular types. J Virol 91: 2457-2466.

34. Shen J, Zhou J, Chen GQ \& Xiu ZL (2018). Efficient genome engineering of a virulent Klebsiella bacteriophage using CRISPR-Cas9. J Virol 92: 00534-18.

35. Yan M, Wen J, Liang M, Lu Y, Kamata M \& Chen IS (2015). Modulation of gene expression by polymer nanocapsule delivery of DNA cassettes encoding small RNAs. PloS One 10: 0127986.

36. Pursey E, Sünderhauf D, Gaze WH, Westra ER \& van Houte S (2018). CRISPR-Cas antimicrobials: challenges and future prospects. PLoS Pathog 14: 6 .

37. Chen S, Liu H, Liang W, Hong L, Zhang B, Huang L \& Duan G (2019). Insertion sequences in the CRISPR-Cas system regulate horizontal antimicrobial resistance gene transfer in Shigella strains. Int J Antimicrob 53: 109-115.

38. De Oliveira Santos IC, Albano RM, Asensi MD \& CarvalhoAAPDA (2018). Draft genome sequence of KPC-2producing Pseudomonas aeruginosa recovered from a bloodstream infection sample in Brazil. $J$ Glob Antimicrob Resist 15: 99-100.

39. Wang C, Wang Y, Zhang L, Miron RJ, Liang J, Shi M \& Zhang Y (2018). Pretreated Macrophage-MembraneCoated Gold Nanocages for Precise Drug Delivery for Treatment of Bacterial Infections. Adv Mater 30: 804023.

40. Hullahalli K, Rodrigues M \& Palmer KL (2017). Exploiting CRISPR-Cas to manipulate Enterococcus faecalis populations. Elife 6: 26664.

41. Ishino $\mathrm{Y}$, Shinagawa $\mathrm{H}$, Makino $\mathrm{K}$, Amemura M \& Nakata A (1987). Nucleotide sequence of the iap gene, responsible for alkaline phosphatase isozyme conversion in Escherichia coli, and identifcation of the gene product. $J$ Bacteriol 169: 5429-33.

42. Mojica FJM, DíezVC, Soria E \& Juez G (2000). Biological signifcance of a family of regularly spaced repeats in the genomes of Archaea, Bacteria and mitochondria. Mol Microbiol 36: 244-266.

43. Hsu, PD, Lander ES \& F Zhang (2014). Development and applications of CRISPR-Cas9 for genome engineering. Cell 157: 1262-1278.

44. Mojica FJM, García MJ \& Soria E (2005). Intervening sequences of regularly spaced prokaryotic repeats derive from foreign genetic elements. J Mol Evol 60: 174-82.

45. Pourcel C, Salvignol G \& Vergnaud G (2005). CRISPR elements in Yersinia pestis acquire new repeats by preferential uptake of bacteriophage DNA, and provide additional tools for evolutionary studies. Micro 151: 653-663.

46. Shabbir MAB, Hao H, Shabbir MZ, Hussain HI, Iqbal Z \& Ahmed S (2016). Survival and evolution of CRiSPR-Cas system in prokaryotes and its applications. Front Immunol 7: 375.

47. Shabbir MAB, Hao H, Shabbir MZ, Wu Q, Sattar A \& Yuan Z (2016). Bacteria vs. bacteriophages: parallel evolution of immune arsenals. Front Microbiol 7: 1.

48. Barrangou R, Fremaux $C$, Deveau $H$, Richards M, Boyaval P \& Moineau S (2007). CRISPR provides acquired resistance against viruses in prokaryotes. Sci 315: 1709-1712.

49. Garneau JE, Dupuis MÈ, Villion M, Romero DA, Barrangou R \& Boyaval P (2011). The CRISPR/Cas bacterial immune system cleaves bacteriophage and plasmid DNA. Nat 468: 67-71.

50. Brouns SJJ, Jore MM, Lundgren $M$, Westra ER, Slijkhuis RJH \& Snijders APL (2008). Small CRISPR RNAs guide antiviral defense in prokaryotes. Sci 321: 960-974.

51. Carte J, Wang R, Li H, Terns RM \& Terns MP (2008). Cas6 is an endoribonuclease that generates guide RNAs for invader defence in prokaryotes. Gen Dev 22: 3489-96.

52. Deveau H, Garneau JE and Moineau S (2010). CRISPR/Cas system and its role in phage-bacteria interactions. Annu Rev 
Microbiol 64: 475-493.

53. Bhaya D, Davison $M$ \& Barrangou $R$ (2011). CRISPR-Cas systems in bacteria and archaea: versatile small RNAs for adaptive defense and regulation. Annu Rev Genet 45: 273-297.

54. Makarova KS, Haft DH, Barrangou R, Brouns SJJ, Charpentier E \& Horvath P (2010) Evolution and classifcation of the CRISPR-Cas systems. Nat Rev Microbiol 9: 467-477.

55. Sinkunas T, Gasiunas G, Fremaux C, Barrangou R, Horvath $\mathrm{P}$ \& Siksnys V (2011). Cas3 is a single-stranded DNA nuclease and ATP-dependent helicase in the CRISPR/Cas immune system. EMBO J 30: 1335-1342.

56. Jore MM, Lundgren M, Van DE, Bultema JB, Westra ER \& Waghmare SP (2011). Structural basis for CRISPR RNA-guided DNA recognition by Cascade. Nat Struct MolBiol 18: 529-536.

57. Lintner NG, Kerou M, Brumfeld SK, Graham S, Liu H \& Naismith JH (2011). Structural and functional characterization of an archaeal clustered regularly interspaced short palindromic repeat (CRISPR)-associated complex for antiviral defense (CASCADE). J Biol Chem 286: 21643-21656.

58. Nam KH, Haitjema C, Liu X, Ding F, Wang H \& Delisa MP (2012). Cas5d protein processes pre-crRNA and assembles into a cascade-like interference complex in subtype IC/Dvulg CRISPRCas system. Str 20: 1574-1584.

59. Wiedenheft B, Van DE, Bultema JB, Waghmare SP, Zhou K \& Barendregt A (2011). RNA-guided complex from a bacterial immune system enhances target recognition through seed sequence interactions. Proc Natl Acad Sci 108: 10092-10097.

60. Deltcheva E, Chylinski K, Sharma CM, Gonzales K, Chao Y \& Pirzada ZA (2011). CRISPR RNA maturation by transencoded small RNA and host factor RNase III. Nat 471: 602-617.

61. Jinek M, Chylinski K, Fonfara I, Hauer M,
Doudna JA \& Charpentier E (2012). A programmable dual-RNA - guided DNA endonuclease in adaptive bacterial immunity. Sci 337: 816-821.

62. Cong L, Ran FA, Cox D, Lin S, Barretto R \& Habib N (2013). Multiplex genome engineering using CRISPR/Cas systems. Sci 339: 819-823.

63. Anantharaman V, Iyer LM \& Aravind L (2010). Presence of a classical RRM-fold palm domain in Thg1-type 3'-5' nucleic acid polymerases and the origin of the GGDEF and CRISPR polymerase domains. Biol Direct 5: 1-9.

64. Zhang J, Rouillon C, Kerou M, Reeks J, Brugger K \& Graham S (2012). Structure and mechanism of the CMR complex for CRISPR-mediated antiviral immunity. Mol Cell 45: 303-313.

65. Deng L, Garrett RA, Shah SA, Peng X \& She Q (2013). A novel interference mechanism by a type IIIB CRISPR-Cmr module in sulfolobus. Mol Microbiol 87: 1088-1099.

66. Singh V, Braddick D \& Dhar PK (2017). Exploring the potential of genome editing CRISPR-Cas9 technology. Gene 599: 118.

67. Bikard D, HatoumAA, Mucida D \& Marraffini LA (2012). CRISPR interference can prevent natural transformation and virulence acquisition during in vivo bacterial infection. Cell Host Microbe 12: 177-186.

68. Yosef I, Manor M, Kiro R \& Qimron U (2015). Temperate and lytic bacteriophages programmed to sensitize and kill antibiotic-resistant bacteria. PNAS 112: 7267-7272.

69. Nierode G, Kwon PS, Dordick JS \& Kwon SJ. (2016). Cell-based assay design for high-content screening of drug candidates. JMB 26: 213.

70. Sommer MO, Church GM \& Dantas G (2010). The human microbiome harbors a diverse reservoir of antibiotic resistance genes. Vit 1: 299-303.

71. Thomas CM \& Nielsen KM (2005). Mechanisms of, and barriers to, horizontal 
gene transfer between bacteria. Nat Rev Microbiol 3: 711-721.

72. Pires DP, Cleto S, Sillankorva S, Azeredo J \& Lu TK (2016). Genetically engineered phages: a review of advances over the last decade. Microbiol Mol Biol Rev 80: 523543.

73. Naduthodi MIS, Barbosa MJ \&Van DOJ (2018). Progress of CRISPR-Cas based genome editing in photosynthetic microbes. Biotechnol J 13: 1700591.

74. Jiang Y, Qian F, Yang J, Liu V, Dong F, Xu C \& R Wang (2017). CRISPR-Cpf1 assisted genome editing of Corynebacterium glutamicum. Nat Commun 8: 151-179.

75. Smith J, Grizot S, Arnould S, Duclert A, Epinat JC, Chames P \& Montoya G (2006). A combinatorial approach to create artificial homing endonucleases cleaving chosen sequences. Nucleic Acids Res 34: 149-149.

76. Miller JC, Tan S, Qiao G, Barlow KA, WangXia DF \& Dulay GP (2011). A TALE nuclease architecture for efficient genome editing. Nat Biotechnol 29: 143.

77. Strong A, \& Musunuru K (2017). Genome editing in cardiovascular diseases. Nat Rev Cardiol 14: 11.

78. Van HS, Ekroth AK, Broniewski JM, Chabas H, Ashby B \& Bondy DJ (2016). The diversity generating benefits of a prokaryotic adaptive immune system. Nat 532(7599): 385-398.

79. Jiang W, Maniv I, Arain F, Wang Y, Levin BR \& Marraffini LA (2013). Dealing with the evolutionary downside of CRISPR immunity: bacteria and beneficial plasmids. PLoS Genet 9(9): e1003844.

80. Vercoe RB, Chang JT, Dy RL, Taylor C, Gristwood T \& Clulow JS (2013). Cytotoxic chromosomal targeting by CRISPR/Cas systems can reshape bacterial genomes and expel or remodel pathogenicity islands. PLoS Genet 9(4): e1003454.

81. Pawluk A, Davidson AR \& Maxwell KL (2018). Anti-CRISPR: discovery, mechanism and function. Nat Rev Microbiol 16(1): $12 \pm 7$.

82. Borges AL, Davidson AR\&BondyDJ (2017). The Discovery, Mechanisms, and Evolutionary Impact of Anti-CRISPRs. Annu Rev Virol 4(1): 37-59.

83. Pawluk A, Staals RH, Taylor C, Watson BN\&Saha S (2016). Fineran PC Inactivation of CRISPR-Cas systems by anti-CRISPR proteins in diverse bacterial species. Nat Microbiol 1(8): 160-185

84. Pawluk A, Amrani N, Zhang Y, Garcia B, HidalgoRY\&Lee J (2016).Naturally Occurring Off-Switches for CRISPRCas9. Cell 167(7): 1829-1838.

85. Harrington LB, Doxzen KW, Ma E, Liu JJ, Knott GJ \& Edraki A (2017). A BroadSpectrum Inhibitor ofCRISPR-Cas9. Cell 170(6): 1224-1233.

86. Spencer SJ, Tamminen MV, Preheim SP, Guo MT, Briggs AW\& Brito IL (2016). Massively parallel sequencing of single cells by epicPCR links functional genes with phylogenetic markers. ISME J 10(2): 427-436.

87. Marbouty M, Baudry L, Cournac A \& Koszul R (2017). Scaffolding bacterial genomes and probing host-virus interactions in gut microbiome by proximity ligation (chromosome capture) assay. Sci Adv 3(2): 1602-1605.

88. Theriot CM, Koenigsknecht MJ, Carlson PE, Hatton GE, Nelson AM \& Li B (2014). Antibiotic-induced shifts in the mouse gut microbiome and metabolome increase susceptibility to Clostridium difficile infection. Nat Commun 5: 3114.

89. Jorth P, Turner KH, Gumus P, Nizam N, Buduneli N \& Whiteley M (2014). Metatranscriptomics of the human oral microbiome during health and disease. $M$ Biol 5(2): 1-15. 